\title{
THE ONSET OF QUASI-PERIODIC VARIATIONS IN Be
}

\section{STARS}

\author{
T. KOGURE and M. MON \\ Department of Astronomy, Faculty of Science, Kyoto University, Sakyo-ku, Kyoto \\ 601-01, Japan \\ and \\ M. SUZUKI \\ Kanazawa Institute of Technology, Kanazawa 921, Japan
}

We present some evidence of the quasi-periodic long-term variations ( $Q P$ $L V)$ in the violet-to-red ratio of double-peaked emission lines $(V / R$ variation) and/or in the radial velocities of shell absorption lines for some Be stars. Although the $V / R$ variations are rather prevailing phenomena among $B e$ stars, the QPLV is remarkable by the following characteristics:

(1) The QPLV appears as a sudden onset of repeated $V / R$ variations after a long (10 years), almost stable period, and it persists for a few or several periods in ten or more years.

(2) The period and amplitude of $V / R$ variations change from cycle to cycle and from star to star. The variations of radial velocities (RV) of shell absorption lines are usually nearly parallel with the $V / R$ variations.

(3) The QPLV appears usually in early type Be stars with large rotational velocities, regardless whether the stars are normal Be or shell stars, and whether they are close-binaries or single stars.

Table I gives a list of Be stars which showed the onset of the QPLV in recent decades. In column 5 , distinction is given for the binarity $(S=$ single, $\mathrm{VB} \& \mathrm{SB}=$ visual and spectroscopic binaries). The X-ray Be star $\gamma$ Cas is supposed to have a compact companion as the $\mathrm{X}$ ray source. Column 6 and 7 give $\bigcirc$ marks when the QPLV is observed in the RV or $V / R$, and column 8 shows the periods of recurrent $V / R$ variations observed in each term of the QPLV. Figure 1 exhibits two examples of the QPLV for $\zeta$ Tau (Ref. 3) and $\gamma$ Cas (Ref. 1)

Two models have so far been proposed on the origin of the $V / R$ variations. One is the rotation-pulsation of the envelope, and the other is the elliptical disk (or ring) model. There is no conclusive arguments as yet on these models, though the elliptical disk model has been found as more preferable in some close binary Be stars by the synchronization of the $V / R$ variation with the orbital motion. The observed behaviors of the QPLV infer that the onset of these phenomena should be originated from some non-linear processes in stars, and taken place as a result of its interaction with the surrounding envelopes. Further studies from observational as well as theretical sides are desirable. 
TABLE I

List of Be Stars that showed Quasi-Periodic Long-Term Variability

\begin{tabular}{|c|c|c|c|c|c|c|c|c|}
\hline \multirow[t]{2}{*}{ Name } & \multirow[t]{2}{*}{ Sp. } & \multirow{2}{*}{$\begin{array}{l}V \sin i \\
\mathrm{~km} / \mathrm{s}\end{array}$} & \multirow{2}{*}{$\begin{array}{l}\mathrm{Be} / \\
\text { Shell }\end{array}$} & \multirow[t]{2}{*}{ Binarity } & \multicolumn{3}{|c|}{ Quasi-Periodic Var. } & \multirow[t]{2}{*}{ Ref. } \\
\hline & & & & & RV & $V / R$ & Quasi-P(yrs) & \\
\hline$\gamma$ Cas & BOIVpe & 300 & $\mathrm{Be}$ & X-ray Be & $\bar{x}$ & 0 & $\mathrm{P}=5,6,7,-$ & 1 \\
\hline $1 \mathrm{H} \mathrm{Cam}$ & $\mathrm{B} 2 \mathrm{Ve}$ & 351 & $\mathrm{~S} / \mathrm{Be}$ & $S B$ & 0 & 0 & $P=6 ?, 6,6,6$ & 2 \\
\hline$\zeta$ Tau & B2IVpe & 310 & $\mathrm{~s}$ & $\begin{array}{l}S B \\
P=132.9 d\end{array}$ & 0 & 0 & $\mathrm{P}=7,4,4, \pi ?$ & 3 \\
\hline$\beta^{1}$ Mon & B3 $\mathrm{Ve}$ & 346 & $\mathrm{~S}$ & $\mathrm{VB}, \mathrm{SB}$ & 0 & 0 & $\mathrm{P} \sim 12.5$ & 4 \\
\hline $48 \mathrm{Lib}$ & B3IIIP & 405 & $\mathrm{~S}$ & $\mathrm{~s}$ & 0 & 0 & $P=9,11,13,6,-$ & 5,6 \\
\hline$\varepsilon$ Cap & B3 Vpe & 293 & $\mathrm{~s}$ & $\mathrm{VB}, \mathrm{SB}$ & $x$ & (O) & $\mathrm{P} \sim 8 ?$ & 7 \\
\hline$\pi$ Aqr & B1 Ve & 278 & $\mathrm{~S}$ & $\mathrm{~s}$ & 0 & 0 & $P=6,2,3,(5),-$ & 8 \\
\hline EW Lac & B2IIIpe & 350 & $\mathrm{~S}$ & $S$ & 0 & 0 & $\mathrm{P} \sim 7$ & 9,10 \\
\hline
\end{tabular}

References:

1. Horaguchi, Kogure, Hirata, Kawai, Matsuoka, Murakami, Doazan, Slettebak, C. C. Huang, Cao, Guo, L. Huang, Tsujita, Ohshima, \& Ito (in preparation).

2. McLaughlin: 1963, ApJ, 137, 1085 .

3. Mon, Kogure, Suzuki, \& Singh,: 1992 PASJ, 44 (in press).

4. Cowley \& Gugula,: 1973, A\&A,, 22, 203.

5. Merrill \& Sanford,: 1944, ApJ, 100, 14.

6. Aydin, \& Faraggiana,: 1978, A\&A, Sup., 34, 51.

7. Mennickent \& Vogt,: 1991, A\&A, 241, 159.

8. McLaughlin,: 1962, ApJ, Sup., 7, 65.

9. Hubert, Floquet et al.: 1987, A\&A. Sup., 70, 443.

10. Kogure \& Suzuki,: 1987, in Physics of Be Stars, eds. Slettebak \& Snow, p. 192. 


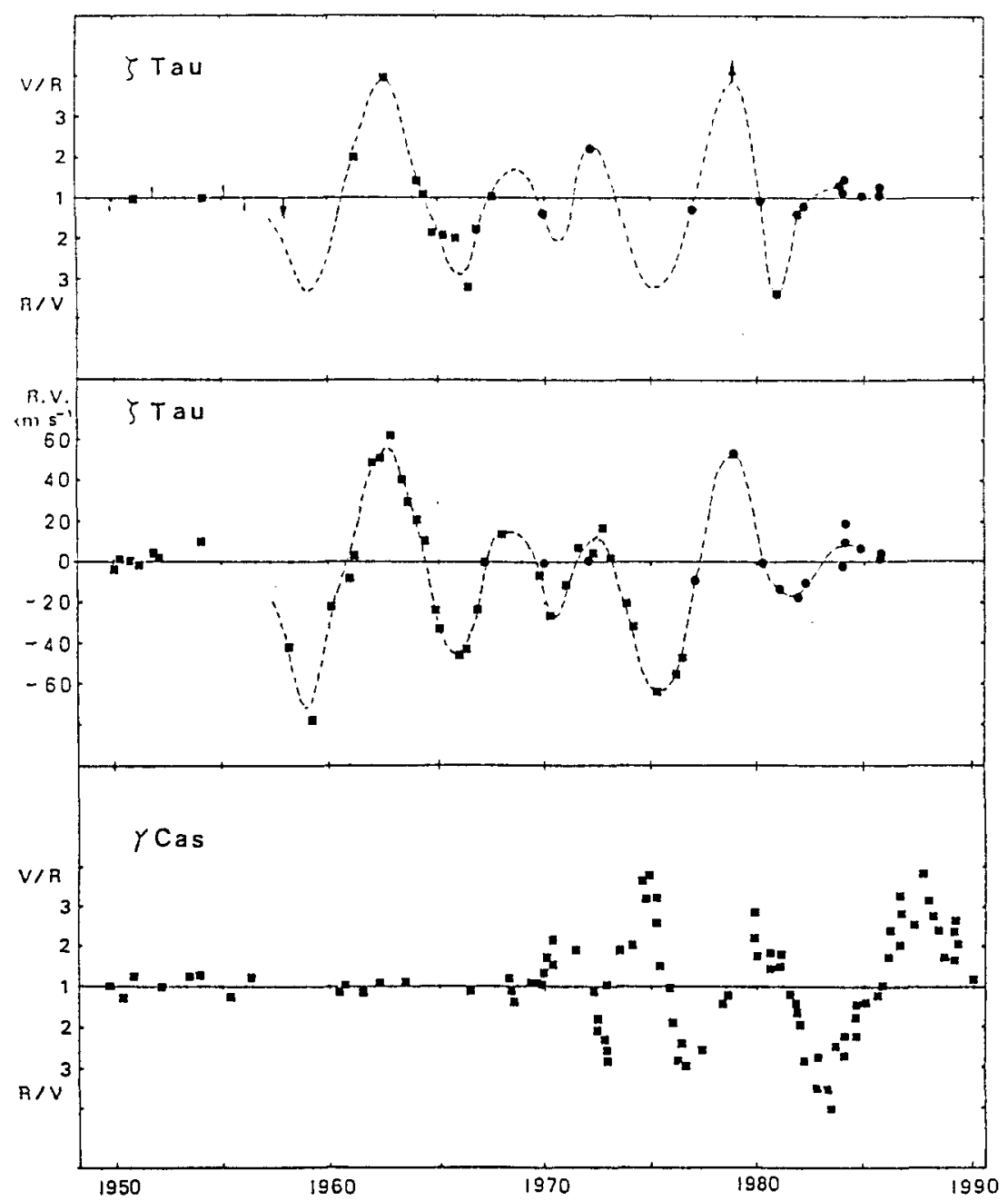

Fig. 1. The QPLV in $\zeta$ Tau (V/R and RV) and $\gamma$ Cas $(V / R)$. Filled circles in $\zeta$ Tau denote our original data (Ref. 3 ). The RV cureve is given after subtracting the binary motion of $\zeta$ Tau. The observational data for $\gamma$ Cas are taken from Horaguchi et al. (Ref. 1). 